\title{
A novel technique for immediate nipple reconstruction with a rectangular flap in implant-based breast reconstruction
}

\author{
Piotr Pluta ${ }^{1}$, Janusz H. Piekarski², Marek Zadrożny ${ }^{1}$
}

\begin{abstract}
${ }^{1}$ Department of Surgical Oncology and Breast Diseases, Polish Mother's Memorial Hospital - Research Institute, Lodz, Poland

2Department of Surgical Oncology, Medical University of Lodz, Lodz, Poland
\end{abstract}

Submitted: 17 May 2021; Accepted: 13 August 2021

Online publication: 25 August 2021

Arch Med Sci

DOI: https://doi.org/10.5114/aoms/141229

Copyright @ 2021 Termedia \& Banach

\section{Abstract}

Introduction: Nipple reconstruction complements breast mound restoration in postmastectomy breast cancer patients. It positively affects patients' welfare, both in psycho-social and sexual aspects. An immediate nipple reconstruction is an alternative approach to delayed surgery. We describe an original technique for an immediate nipple reconstruction using a modified rectangular flap.

Material and methods: One hundred and seventeen nipple reconstructions in 112 breast cancer patients were performed. This technique was used during skin-sparing mastectomy, including 104 (88.9\%) implants and 13 (11.1\%) tissue-expander breast reconstructions. Synthetic meshes covered with titanium supported lower breast poles in 72 (61.5\%) implantations; in the remaining cases $(38.5 \%)$, muscles covered the entire implant. Preoperative chemotherapy was applied in $18.75 \%$ of the patients; adjuvant chemo- and hormonal therapy were applied in $29.5 \%$ and $74.1 \%$ of the patients, respectively. Twenty-four (21.4\%) patients were irradiated postoperatively.

Results: In 5 (4.3\%) out of 117 procedures, necrosis of the rectangular flap was observed. Twelve months after surgery, 2 (1.7\%) cases of loss of projection of the reconstructed nipple were reported. In the twelfth month of observation, of the subgroup of 102 patients with permanent nipple presentation, $93.1 \%$ gave a positive opinion regarding the procedure.

Conclusions: The applied immediate nipple reconstruction technique performed during a skin-sparing mastectomy and implant/expander-based breast reconstruction was an effective and safe adjunct curative breast surgery.

Key words: implants, breast, immediate nipple reconstruction.

\section{Introduction}

A nipple reconstruction complements breast mound restoration in postmastectomy breast cancer patients. Thus, the breast regains a natural look; it positively affects patients' welfare, both in psychosocial and sexual aspects [1]. In most cases, reconstruction of the nipple or the nipple-areola complex (NAC) is performed as a separate and final breast reconstruction stage. Numerous delayed nipple reconstruction techniques proved their feasibility, leading to permanent and satisfactory cosmetic outcomes [2].

\author{
Corresponding author: \\ Piotr Pluta PhD \\ Department of \\ Surgical Oncology \\ and Breast Diseases \\ Polish Mother's \\ Memorial Hospital - \\ Research Institute, \\ Lodz, Poland \\ Phone: +48 604188815 \\ E-mail: drpiotrpluta@gmail. \\ com
}


Delayed nipple reconstruction using skin flaps is, however, not free from drawbacks. In patients who have undergone previous surgery or postoperative radiotherapy and may have thin or atrophic skin flaps, nipple reconstruction may be challenging. Failure of nipple reconstruction may even lead to breast implants' exposure [3]. Therefore, an apprehension of the potential risk of complications often prolongs the interval between the breast mound's and the nipple reconstructions, which may deter patients from this procedure [4].

An alternative approach to delayed surgery for breast cancer patients is immediate nipple reconstruction. Several techniques have been presented for performing such types of reconstructions, both in autologous and implant-based breast reconstructions [5-10]. However, the tailoring strategy for individual patients and the expectations for rapid aesthetic successes still power breast surgery evolution.

Table I. Characteristics of the patients (112 breast cancer patients; 117 skin-sparing mastectomies (SSM) with breast and nipple reconstruction; one type of adjuvant treatment does not exclude the use of another one)

\begin{tabular}{|c|c|}
\hline Characteristic & Results \\
\hline Mean age (range) [years] & $57(35-79)$ \\
\hline \multicolumn{2}{|l|}{ Cancer stage, $n(\%)$ : } \\
\hline 0 (DCIS) & $11(9.8)^{\star}$ \\
\hline I & $46(41.1)^{\star}$ \\
\hline II & $51(45.5)^{\star}$ \\
\hline III & $4(3.6)^{*}$ \\
\hline \multicolumn{2}{|l|}{$\begin{array}{l}\text { SSM with breast and nipple } \\
\text { reconstruction, } n(\%) \text { : }\end{array}$} \\
\hline Unilateral & $107(95.5)^{*}$ \\
\hline Bilateral & $5(4.5)^{\star}$ \\
\hline \multicolumn{2}{|l|}{ Breast reconstruction, $n(\%)$ : } \\
\hline Implant & $104(88.9)^{\star *}$ \\
\hline Expander/implant & $13(11.1)^{\star *}$ \\
\hline \multicolumn{2}{|l|}{ Implant/expander cover, $n(\%)$ : } \\
\hline Pectoralis major muscle & $45(38.5)^{\star *}$ \\
\hline $\begin{array}{l}\text { Pectoralis major muscle and synthetic } \\
\text { mesh }\end{array}$ & $72(61.5)^{\star *}$ \\
\hline \multicolumn{2}{|l|}{ Surgery on axillary lymph nodes, $n(\%)$ : } \\
\hline Sentinel node biopsy (alone) & $77(65.8)^{\star *}$ \\
\hline $\begin{array}{l}\text { Axillary lymph node dissection } \\
\text { (cN1 or pN1sn) }\end{array}$ & $40(34.2)^{\star *}$ \\
\hline \multicolumn{2}{|l|}{ Adjuvant/neoadjuvant treatment, $n(\%)$ : } \\
\hline Radiotherapy & $24(21.4)^{\star}$ \\
\hline Neoadjuvant chemotherapy & $21(18.75)^{\star}$ \\
\hline Adjuvant chemotherapy & $33(29.5)^{*}$ \\
\hline Hormone therapy & $83(74.1)^{*}$ \\
\hline
\end{tabular}

Herein, we describe our experiences with immediate implant-based breast reconstruction modified by restoring the nipple with a rectangular skin flap. In contrast to the former techniques, our method is simple as it directly adapts the conventional elliptical breast incision.

\section{Material and methods}

A study was conducted between June 2015 and December 2020 at the $1^{\text {st }}$ and $2^{\text {nd }}$ Department of Surgical Oncology, Copernicus Memorial Hospital in Lodz and the Department of Surgical Oncology and Breast Diseases, Polish Mother's Memorial Hospital - Research Institute, Lodz. A total of 117 one-stage nipple reconstructions were performed in 112 breast cancer patients undergoing skin-sparing mastectomy (SSM). The patients gave informed consent for the surgery, including nipple reconstruction. The authors of the study performed all breast and nipple reconstructions. The Bioethics Committee of the District Medical Council in Lodz accepted the study protocol.

The mean age of the patients was 57 years (range: 35-79 years). Anatomic gel implants (Allergan Natrelle 410 or Mentor CPG) were used in direct-to-implant breast reconstruction in 104 (88.9\%) procedures. Two-stage tissue expander/ implant reconstruction (Mentor CPX4/Mentor CPG) was applied in 13 (11.1\%) cases. Synthetic meshes covered with titanium (TiLOOP Bra, pfm medical) supported the lower breast poles in $72(61.5 \%) \mathrm{im}$ plantations; in the remaining cases (38.5\%) muscles covered the entire implant. No pre-pectoral breast reconstruction was performed in this group.

During 117 procedures, 112 (95.7\%) sentinel lymph node biopsies (SLNB) were carried out. Metastasis-free sentinel nodes were confirmed in 77 cases; in the remaining 35 biopsies, axillary lymph node dissection (ALND) complemented the surgery due to metastatic sentinel nodes. In 5 (3.4\%) patients, selective ALND was performed due to the presence of clinically positive lymph nodal metastases (cN1).

The clinical stage of the disease was classified according to the $7^{\text {th }}$ edition of the Union for International Cancer Control TNM Classification of Malignant Tumors [11]. Preoperative chemotherapy was applied in $18.75 \%$ of the patients; adjuvant chemo- and hormonal therapy were applied in $29.5 \%$ and $74.1 \%$ of the patients, respectively. Twenty-four $(21.4 \%)$ patients were irradiated postoperatively. Characteristics of the patients are presented in Table I.

Early postoperative complications, reported within six months after the surgery, included: hematoma, wound infection, implant loss, and skin flap necrosis. The observed skin flap necrosis was subdivided into isolated necrosis of the re- 
constructed nipple (up to $2 \mathrm{~cm}$ from the tip of the nipple) and necrosis of the wound edges and the nipple (necrosis exceeding $2 \mathrm{~cm}$ from the base of the nipple).

According to the study protocol, all patients were examined 12 months after the surgery. We recorded patients' breast complaints and defined them as midterm complications. To assess the patients' satisfaction with the nipple reconstruction, we used a 4-point Likert-like scale; the patients answered the question: "How satisfied are you with the result of the nipple reconstruction?", choosing one of the following options: 1 - very
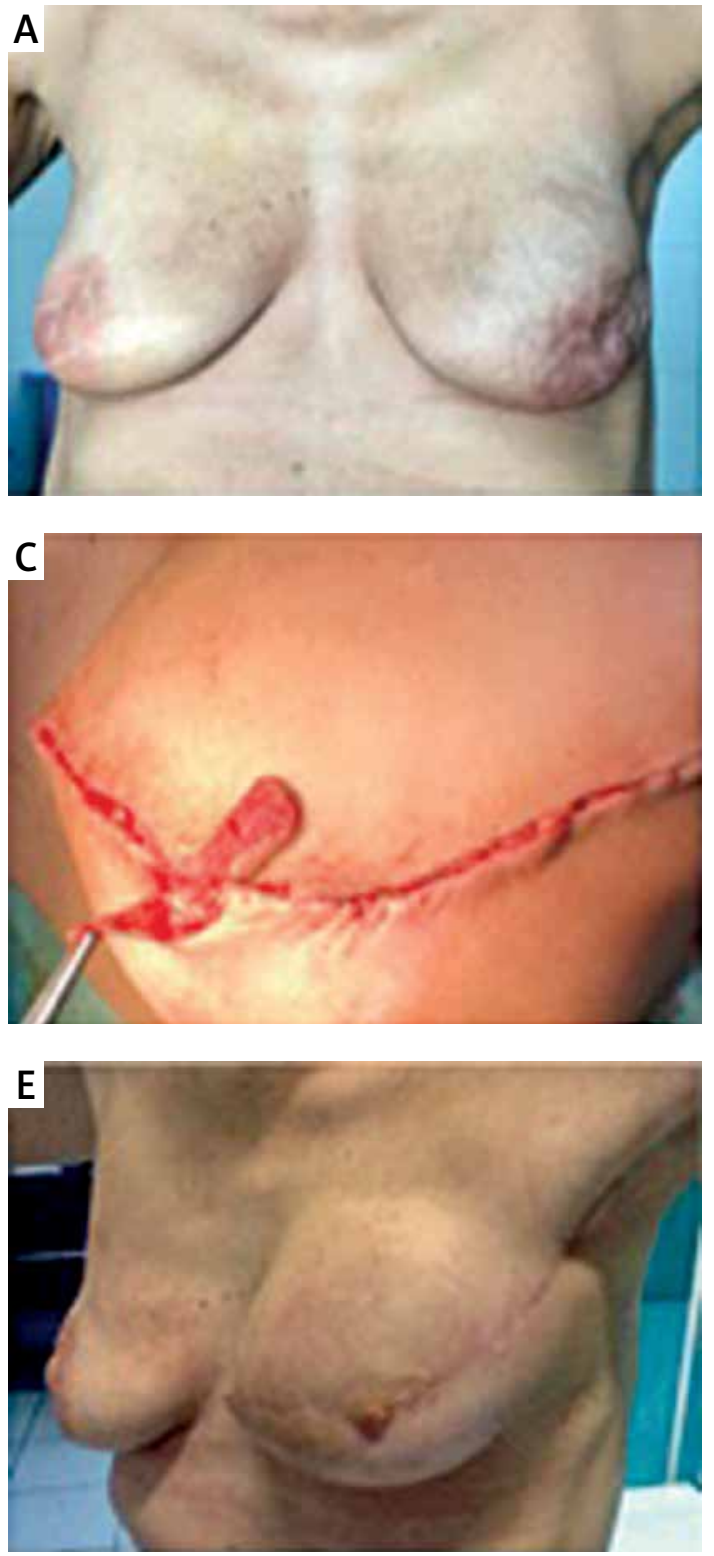

unsatisfied; 2 - unsatisfied; 3 - satisfied; 4 - very satisfied.

\section{Surgical technique}

The skin incision lines, including the rectangular flap for nipple reconstruction, were planed in the patients standing position before surgery. Mastectomy incisions were outlined symmetrically to the NAC to determine the optimal location for the reconstructed nipple. The range of skin removal relates to the characteristics of the primary tumour and the shape of the breast (Figures $1 \mathrm{~A}, \mathrm{~B}$ ).
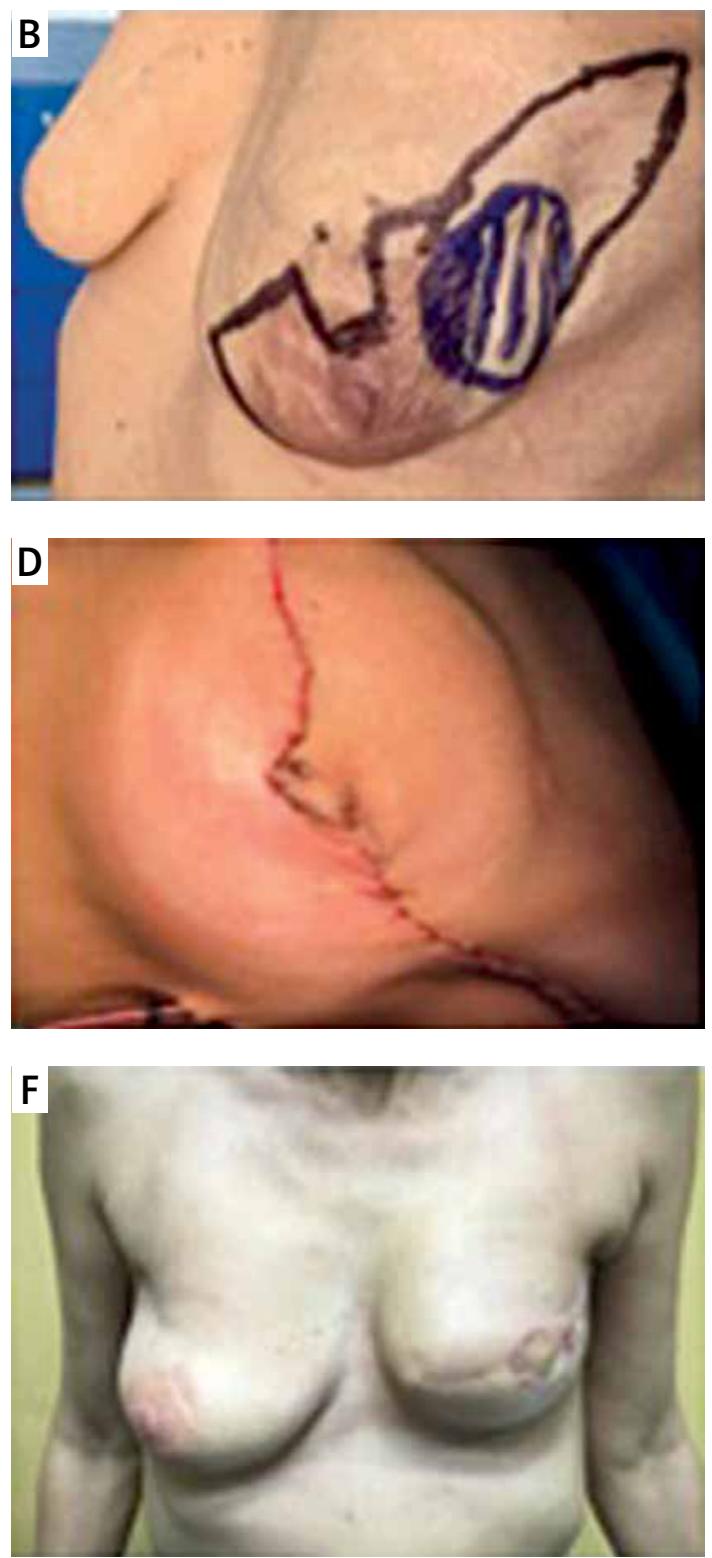

Figure 1. A - A 47-year-old patient was diagnosed with recurrent left breast cancer (rT2NOMO) 3 years after breast conservative treatment. B - She was offered a skin-sparing mastectomy with sentinel node biopsy and an immediate breast and nipple reconstruction (expander, $350 \mathrm{ml}$ ). C - For nipple restoration, rectangular flap based on the caudal mastectomy skin flap pedicle was elevated. D - On the opposite edge of the wound, a small portion of the skin was de-epithelialized to prepare the place for nipple flap fixation. E - The expander was filled-up with saline during the next 3 months, and then replaced for the definitive implant $(215 \mathrm{~g})$. The areola was reconstructed with two semi-circular thin skin flaps. F - Figure presents the outcome in the 12-month of follow-up 
The rectangular flap was optionally made from the upper or lower edge of the skin incision. The flap base was about 15-20 mm long, and its height was between 30 and $40 \mathrm{~mm}$. If an extensive resection of breast skin was not required, the flap was made from the areola skin, so the new nipple achieved a natural brownish colour. Care was taken to choose the proper mastectomy flap's thickness as the subcutaneous vascular plexus preservation does not impair the total glandular breast tissue resection. Depending on the surgeon' preferences, electrosurgery or scissors dissection was used for the elevation of flaps. The generator's standard breast surgery settings were applied with low to medium power (mode fulguration; effect 3-4; power 40-60 watts).

Preparation of the skin flaps, removal of the breast, and an implant or expander insertion under the pectoralis major muscle did not deviate from the standard procedures of immediate breast reconstruction. If necessary, we used synthetic meshes to cover the implant in the lower breast quadrants (TiLOOP Bra). SLNB or ALND was performed using the same or separate incisions. Two suction drains were placed in the wound (tube size 12 to 18 French) and main- tained until the fluid collection was less than $20 \mathrm{ml} /$ day.

Except for the reconstructed nipple, the wound was closed with two layers of continuous, absorbable sutures (Monocryl 3-0 and 4-0). The rectangular flap was folded in half by stitching the flap's side edges with non-absorbable sutures (Prolene 5-0). The flap's distal edge was stitched to the opposite border of the wound. A crescent de-epithelisation was performed to make a place for attachment of the flap's free edge (Figures 1 C, D).

Areola reconstruction was performed using a tattoo, free skin graft or rotated flap, not earlier than 6 weeks after the surgery (Figures 1 E, F). Figure 2 schematically depicts the principles of the method.

\section{Results}

Isolated necrosis of the rectangular flaps was observed in 5 (4.3\%) cases; none required surgical debridement. Among 8 cases of necrosis of the wound edges and the nipple $(8 / 117 ; 6.8 \%)$, $2(1.7 \%)$ cases resulted in implant loss. Surgical site infection was reported in 9 (7.7\%) out of 117 procedures; the inflammation subsided after an-
A

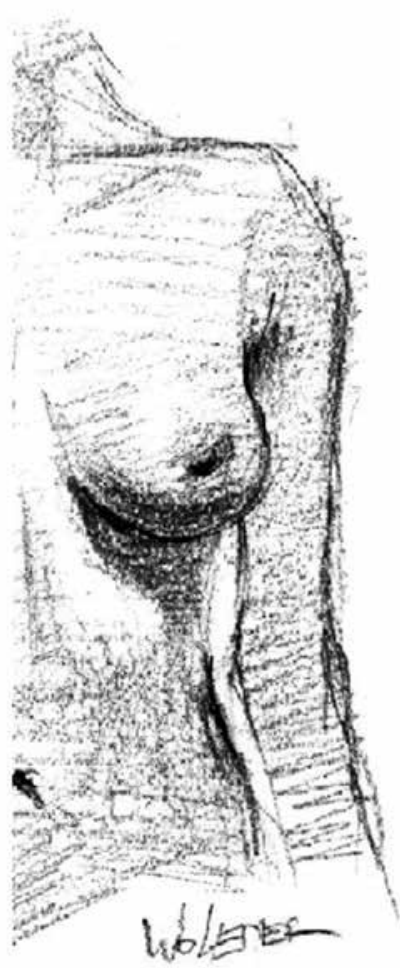

B

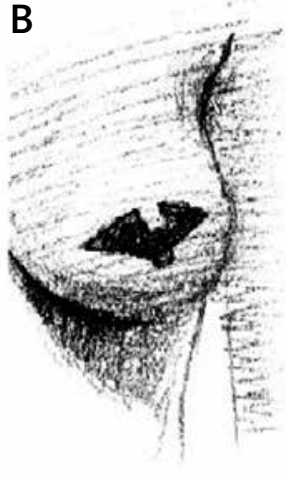

C

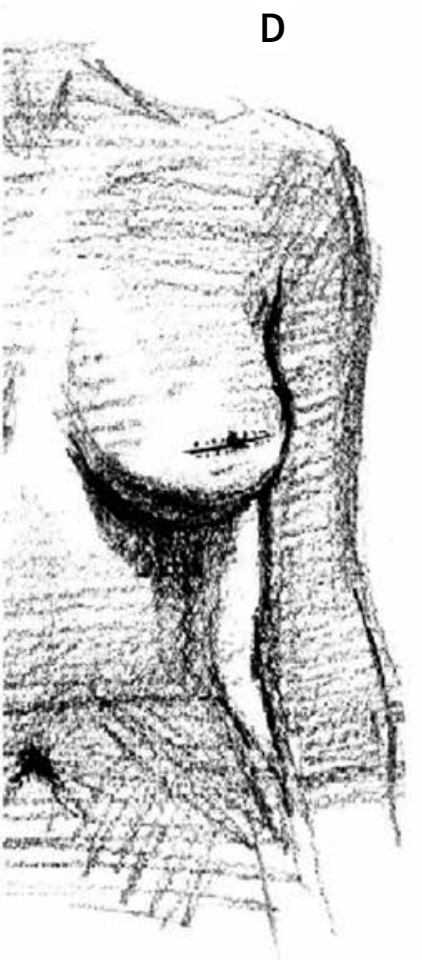

Figure 2. A - While preoperatively drawing, patients were in a standing position. B - The rectangular flap was optionally made from the upper or lower edge of the skin incision. C - The flap base was about 15-20 mm in length, and its height between $30-40 \mathrm{~mm}$. The area in the opposite border to the flap will further undergo de-epithelialization. After the mastectomy and the implant/expander breast reconstruction, the wound was closed, excluding the nipple flap location. D - The rectangular flap was folded in half, and the flap's side edges were stitched. The flap's distal edge was attached to the outer border of the area of de-epithelialization. As mastectomy incisions are outlined symmetrically to the NAC, it defines the optimal location for the reconstructed nipple 
tibiotic therapy. In 4 (3.4\%) cases, postoperative bleeding was an indication for wound revision.

One year after surgery, 5 (4.3\%) patients reported discomfort associated with capsular contracture formation. Total loss of projection was observed for 2 (1.7\%) reconstructed nipples. Early and midterm surgical outcomes are summarised in Table II. An example of a patient after immediate breast and nipple reconstruction is presented in Figure 3.

Twelve months after surgery, patients assessed their satisfaction with the cosmetic effect of nipple reconstruction. Of the subgroup of 102 patients with permanent nipple presentation, 93.1\% gave a positive opinion regarding the procedure (Table III).

\section{Discussion}

The presented technique encompasses two standard surgical procedures: the rectangular flap for nipple reconstruction and the elliptical skin incision for mastectomy. Dini and Ferreira previously described the rectangular flap in patients after complete NAC necrosis in implant-reconstructed breasts [12]. This approach required 3-step surgery with primary free graft coverage of the cicatrix, partial elevation of the rectangular flap improving its vascularity, and final raising of the flap and shaping the nipple. In contrast, we adopted the rectangular flap in immediate nipple reconstruction, so we worked on unharmed tissue. A relatively broad base of the rectangular flap further provided a sufficient blood supply to the formed flap, which resulted in a low rate of isolated nipple necrosis (4.2\%). The effect of such nipple reconstruction was also permanent, as loss of flap projection was reported only in 2 patients.

Similarly, in implant-based breast reconstruction, Highton and Murphy presented an example of a high-efficient technique for immediate nipple

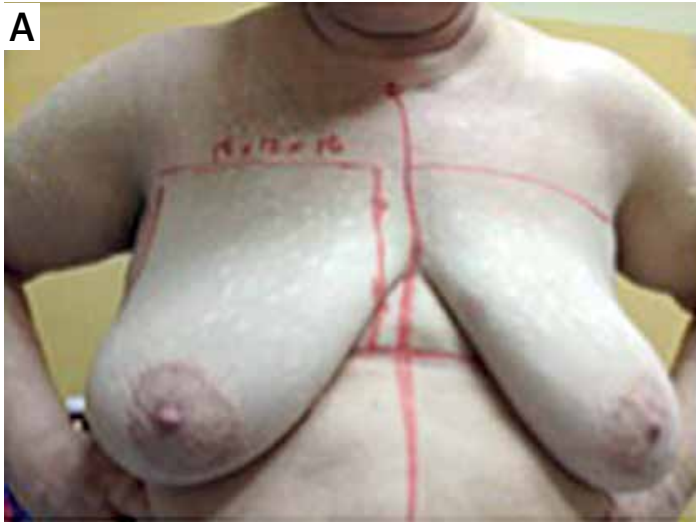

Figure 3. A - 58-year-old patient after neoadjuvant chemotherapy administered due to locally advanced breast cancer. B - Skin-sparing mastectomy with axillary lymph nodes dissection was performed. Simultaneously breast and nipple reconstruction using a rectangular flap was performed. An implant (625 g) was covered with the major pectoralis muscle and a synthetic mesh. After the surgery, the patient was irradiated to the breast and axillary/ supraclavicular lymph nodes - the appearance of the breast and the nipple in 12 months follow-up

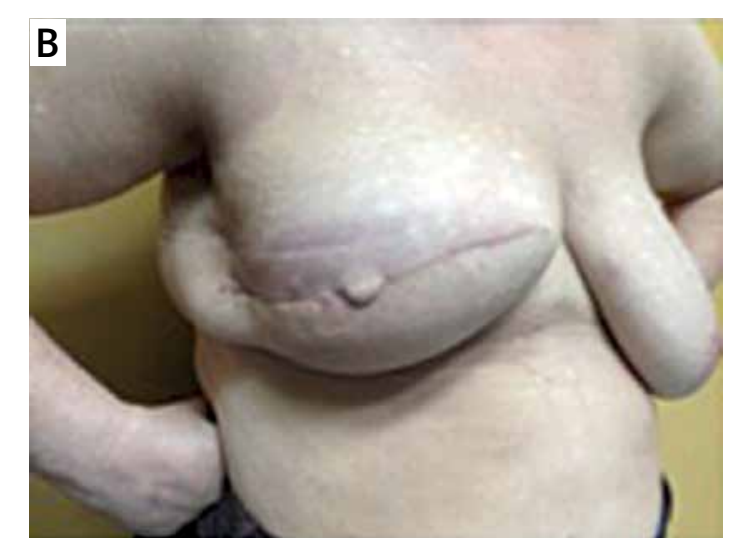

Table II. Complications after skin-sparing mastectomy and immediate reconstruction of the breast and the nipple (117 procedures)

\begin{tabular}{|ll|}
\hline Complications & $\boldsymbol{N}(\%)$ \\
\hline Early: & $5(4.3)$ \\
\hline Isolated nipple necrosis & $8(6.8)$ \\
\hline Nipple and skin flaps necrosis & $2(1.7)$ \\
\hline Skin infection & $4(3.4)$ \\
\hline Hematoma requiring revision & $9(7.7)$ \\
\hline Midterm (12 months after surgery): & $4(3.4)$ \\
\hline Loss of nipple projection & $2(1.7)$ \\
\hline Symptomatic capsule fibrosis & $5(4.3)$ \\
\hline
\end{tabular}

reconstruction [10]. They successfully used a C-V flap and skin graft for nipple-areola reconstruction in 32 cases, with only one partial necrosis of the areola. Hong et al., likewise, successfully used a modified C-V flap ("boomerang flap") in immediate breast and nipple reconstruction in implant-based surgery with no differences between total or partial loss of the nipple in primary and secondary nipple reconstructions [9].

The elliptical skin incision facilitates breast resection and ensures the reconstructed nipple's proper location. We used this incision in all patients regardless of breast size. Our method resulted in $6.8 \%$ necrosis of mastectomy flaps. This complication further affected the rate of nipple reconstruction failure and, in 2 patients, led to implant loss $(1.7 \%)$. The above rate of skin flap necrosis is not negligible, but still lower than the results of the other types of SSM commonly used in medium- and large-breasted women. Carlson et al. assessed skin flap necrosis risk after skin-sparing mastectomy from $10 \%$ to $22 \%$, with the highest risk of complications in SSM type IV 
Table III. Patient self-evaluation of cosmetic satisfaction with the reconstructed nipple 12 months after surgery (102 patients with permanent results of the nipple reconstruction with rectangular flap)

\begin{tabular}{|lc|}
\hline Score & $\boldsymbol{N}(\%)$ \\
\hline 1 - very unsatisfied & $2(2)$ \\
\hline $2-$ unsatisfied & $5(4.9)$ \\
\hline $3-$ satisfied & $53(52)$ \\
\hline $4-$ very satisfied & $42(41.2)$ \\
\hline
\end{tabular}

(Wise pattern) [13]. Santanelli et al. noted a frequency of skin flap ischemia as high as $26.6 \%$ among 75 Wiese pattern SSM, with a $4 \%$ implant loss rate [14].

Rancati et al. suggest evaluating preoperative mammograms to establish the optimal thickness of the flap and reconstruction approach (implant vs expander) to reduce skin necrosis in immediate breast reconstruction and, consequently, implantation failure [15]. We have selectively implemented this procedure, especially in slim patients. Nonetheless, we believe that the atraumatic operative technique and identification of anatomical dissection planes are crucial in avoiding flap necrosis and implant loss.

Multidisciplinary breast collaboration has overturned the dogma that expected radiotherapy to be a contraindication for immediate implant-based reconstruction; still, implant irradiation increases the risk of implant loss and development of symptomatic capsule fibrosis $[16,17]$. In the present study, one-fifth of patients were postoperatively irradiated. Capsule fibrosis occurred in 5 out of 24 patients after breast irradiation, and we reported no implant loss. No patient from this subgroup had nipple flattening, which confirms Jung et al.'s observation of the limited impact of irradiation on the new nipples; this hypothesis requires confirmation in a larger sample of patients [8].

Twelve months after surgery, over $90 \%$ of patients favourably assessed the cosmetic outcome of the restored nipple. In our opinion, this result demonstrates that immediate nipple reconstruction is a reasonable adjustment to breast mound reconstruction. Likewise, Jung et al. reported that $77 \%$ of patients scored the outcome of primary nipple-areola complex (NAC) reconstruction with C-V flap and skin graft as excellent or high [8]. Although Nedomansky et al. reported greater patient satisfaction after delayed reconstruction of the NAC than immediate replantation, we consider that no primary nipple reconstruction method restricted further cosmetic improvements, e.g., non-invasive 3D tattooing [18].

We presented the results of the nipple reconstruction selectively in subpectoral breast reconstruction. We are currently introducing this technique in pre-pectoral breast reconstruction; however, this group of patients is too small to draw conclusions. Another limitation of the study is its lack of comparison with the outcomes of delayed nipple reconstructions. In our practice, patients seldom chose to have delayed NAC reconstructions, which, incidentally, affected our decision to perform all-in-one surgery.

In conclusion, we have presented the method of immediate nipple reconstruction with the rectangular flap. While this technique is a simple adjustment to the conventional elliptical incision employed in skin-sparing mastectomy, it may be a reasonable option against delayed nipple reconstructions, shortening the whole breast restoring process.

\section{Acknowledgments}

We thank Prof. Wojciech Leder from Strzeminski Academy of Art, Lodz, Poland, for preparing the drawings (Figure 2).

\section{Conflict of interest}

The authors declare no conflict of interest.

\section{References}

1. Bykowski MR, Emelife PI, Emelife NN, et al. Nipple-areola complex reconstruction improves psychosocial and sexual well-being in women treated for breast cancer. J Plast Reconstr Aesthet Surg 2017; 70: 209-14.

2. Farhadi J, Maksvytyte GK, Schaefer DJ, et al. Reconstruction of the nipple-areola complex: an update. J Plast Reconstr Aesthet Surg 2006; 59: 40-53.

3. Momeni A, Ghaly M, Gupta D, et al. Nipple reconstruction: risk factors and complications after 189 procedures. Eur J Plast Surg 2013; 36: 633-8.

4. Losken A, Duggal CS, Desai KA, et al. Time to completion of nipple reconstruction: what factors are involved? Ann Plast Surg 2013; 70: 530-2.

5. Delay E, Mojallal A, Vasseur C, et al. Immediate nipple reconstruction during immediate autologous latissimus breast reconstruction. Plast Reconstr Surg 2006; 118: 1303-12.

6. Lee S, Jung Y, Bae Y. Immediate nipple reconstruction as oncoplastic breast surgery: the cigar roll flap with inner dermal core technique. Aesthetic Plast Surg 2015; 39: 706-12.

7. Hyza P, Streit L, Vesely J, et al. New technique of immediate nipple reconstruction during immediate autologous DIEP or MS-TRAM breast reconstruction. Ann Plast Surg 2015; 74: 645-51.

8. Jung $Y$, Lee J, Lee $S$, Bae Y. Immediate nipple reconstruction with a C-V flap and areolar reconstruction with an autograft of the ipsilateral areola. ANZ J Surg 2017; 87: E300-4.

9. Hong KY, Kim YE, Minn KW, et al. Immediate nipple reconstruction during implant-based breast reconstruction. Aesthetic Plast Surg 2017; 41: 793-9.

10. Highton LR, Murphy JA. Immediate nipple-areolar complex Reconstruction for patients undergoing implant-based reconstruction or therapeutic mammoplasty. Plast Reconstr Surg Glob Open 2017; 5: e1243. 
11. Sobin LH, Gospodarowicz MK, Wittekind C. TNM Classification of Malignant Tumours. $7^{\text {th }}$ ed. Wiley-Blackwell 2009.

12. Dini GM, Ferreira LM. The graft said, "when I grow up, I want to be a flap": nipple reconstruction with the graft flap. Plast Reconstr Surg 2006; 117: 333-4.

13. Carlson GW. Technical advances in skin sparing mastectomy. Int J Surg Oncol 2011; 2011: 396901.

14. Santanelli F, Longo B, Sorotos M, et al. Flap survival of skin-sparing mastectomy type IV: a retrospective cohort study of 75 consecutive cases. Ann Surg Oncol 2013; 20: 981-9.

15. Rancati AO, Angrigiani CH, Hammond DC, et al. Direct to implant reconstruction in nipple sparing mastectomy: patient selection by preoperative digital mammogram. Plast Reconstr Surg Glob Open 2017; 5: e1369.

16. Pu Y, Mao TC, Zhang YM, et al. The role of postmastectomy radiation therapy in patients with immediate prosthetic breast reconstruction: a meta-analysis. Medicine 2018; 97: e9548.

17. Kaidar-Person O, Vrou Offersen B, et al. ESTRO ACROP consensus guideline for target volume delineation in the setting of postmastectomy radiation therapy after implant-based immediate reconstruction for early stage breast cancer. Radiother Oncol 2019; 137: 159-66.

18. Nedomansky J, Nickl S, Maier B, et al. Management of the nipple-areola complex in selected patients undergoing primary breast reconstruction: a comparison of immediate replantation and delayed reconstruction. Ann Plast Surg 2017; 78: 379-85. 